Received: 21 May 2018

Accepted: 6 August 2018

Published online: 23 August 2018

SCIENTIFIC REP

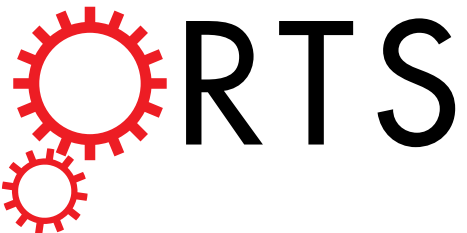

\title{
OPEN
}

\section{Impact of Side Chain Polarity on Non-Stoichiometric Nano- Hydroxyapatite Surface Functionalization with Amino Acids}

\section{Patricia Comeau \& Thomas Willett}

In this study the affinity of three amino acids for the surface of non-stoichiometric hydroxyapatite nanoparticles ( $\mathrm{ns}-\mathrm{nHA}$ ) was investigated under different reaction conditions. The amino acids investigated were chosen based on their differences in side chain polarity and potential impact on this surface affinity. While calcium pre-saturation of the calcium-deficient ns-nHA was not found to improve attachment of any of the amino acids studied, the polarity and fraction of ionized functional side groups was found to have a significant impact on this attachment. Overall, amino acid attachment to ns-nHA was not solely reliant on carboxyl groups. In fact, it seems that amine groups also notably interacted with the negative ns-nHA surface and increased the degree of surface binding achieved. As a result, glycine and lysine had greater attachment to ns-nHA than aspartic acid under the reaction conditions studied. Lastly, our results suggest that a layer of each amino acid forms at the surface of ns-nHA, with aspartic acid attachment the most stable and its surface coverage the least of the three amino acids studied.

The mechanical properties, biocompatibility, and osteoconductive properties of hydroxyapatite (HA) alone has led to extensive research towards the use of this material in biomedical and industrial applications such as in orthopaedics ${ }^{1,2}$. Non-stoichiometric carbonated hydroxyapatite in particular is garnering increased attention for use in biomedical applications owing to its greater chemical similarity to the mineral phase of bone (compared to stoichiometric hydroxyapatite $)^{3}$. By taking advantage of the adsorption properties of nHA, the surface chemistry of these nanoparticles may be tuned by using a functionalization approach with water-soluble biomolecules. In this study, we report on the binding of different amino acids to a non-stoichiometric carbonated nHA (ns-nHA) surface using different reaction conditions.

Amino acids have previously been proposed as ideal candidates for such an approach as a result of their amphoteric nature and ability to interact with HA surfaces, as well as their low cost and intrinsic biocompatibility ${ }^{1,4}$. Prior studies have largely focused on the effect of amino acids on HA crystallization, with negatively charged amino acids (e.g. aspartic acid) commonly being shown to control HA mineralization in bone most effectively ${ }^{5-9}$. However, there are contradictory results reported in the literature on the interaction of amino acids with HA, such that a comprehensive understanding of the mechanisms involved remains largely elusive. For example, Jack et al. ${ }^{2}$ reported that a positively charged amino acid had the strongest affinity for the HA surface, while in a series of papers Koutsopoulos and Dalas ${ }^{5-8,10}$ reported that the largest affinity was with a negatively charged amino acid (specifically aspartic acid). More recent studies have begun to emphasize the impact of side chain functionalities and how interactions at the surface of stoichiometric HA may be maximized as a result ${ }^{9,11,12}$. For example, in a simulated modelling study, Rimola et al. ${ }^{11}$ reported that, as a result of side chain interactions with HA, Lysine and other investigated polar amino acids had a greater surface stability than non-polar glycine. How such findings may apply to ns-nHA remains inconclusive and more investigation is necessary. To address these inconsistencies, this study was conducted to further our understanding of amino acid affinity with the ns-nHA surface and to further realize any side group dependence on this affinity.

Composite Biomaterial Systems Laboratory, Department of Systems Design Engineering, University of Waterloo, Waterloo, ON, N2L 3G1, Canada. Correspondence and requests for materials should be addressed to T.W. (email: thomas.willett@uwaterloo.ca) 


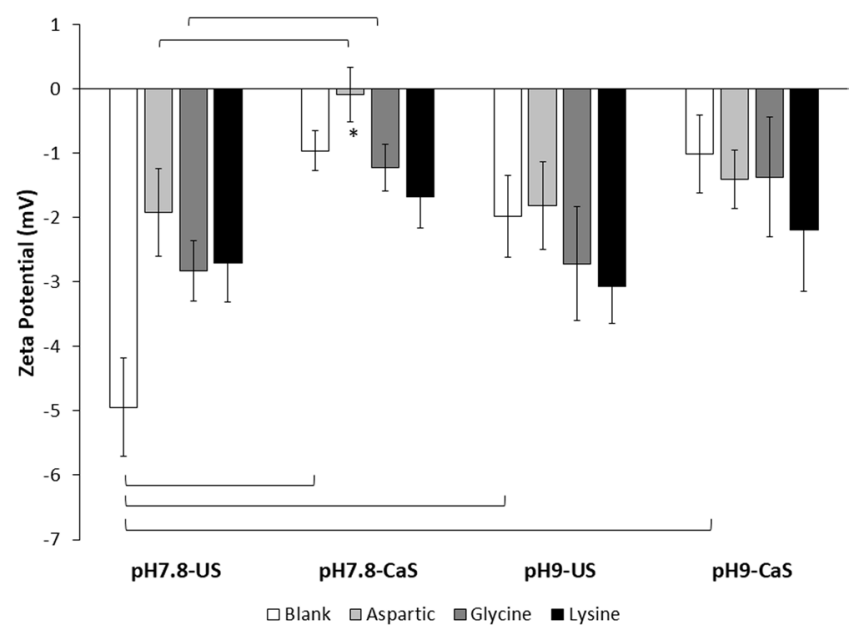

Figure 1. Zeta potential analysis of functionalized ns-nHA. Pre-conditioning of blank ns-nHA and addition of any of the amino acids studied notably increased the zeta potential (i.e. made it less negative). Of particular note is the near-neutral zeta potential of aspartic acid functionalized ns-nHA prepared under calcium-saturated and $\mathrm{pH} 7.8$ conditions (indicated by *). This balanced charge likely results from a more balanced binding of both carboxyl and amine groups to the ns-nHA surface (compared to the other amino acids). Data reported as average \pm one standard deviation $(\mathrm{n}=5)$. ns-nHA unsaturated with calcium is given as US, while that presaturated with calcium is given as CaS. Horizontal bars indicate statistically detectable difference $(\mathrm{p}<0.05)$.

Unfortunately, functionalizing nHA utilizing the more traditional crystallization approach has been found to interfere with ion attachment on certain crystal faces and to create additional defects or inconsistencies in the HA crystal structure, as well as lead to reduced crystallinity ${ }^{13-16}$. Intrinsic factors such as defect density are known to reduce material strength ${ }^{17}$, and could compromise the mechanical performance of not only the mineral phase (e.g. nHA), but ultimately any future composite. Owing to our interest in pursuing composite development with the functionalized ns-nHA, this study sought the development of a more direct approach to functionalizing the surface of ns-nHA.

Hydrogen bonding, as well as electrostatic and van der Waals interactions between amino acids and nHA govern the adsorption of amino acids onto the nHA surface. Analysis of the electrostatic potential at the HA surface has previously revealed a highly positive potential on top of calcium ions and deep negative zones in the proximity of oxygen atoms of the $\mathrm{PO}_{4}$ groups ${ }^{11}$. As a result, the strength of interactions is primarily dependent on such surface properties of nHA, but may also be influenced by experimental conditions including $\mathrm{pH}$ of the medium and type of amino acid $^{18}$. For example, as a result of the different side chain groups, each amino acid possesses a different isoelectric point ( $\mathrm{pI}$ ) and, hence, unique net surface charge ${ }^{4}$. In this study, we investigated the affinity of three different amino acids for the surface of ns-nHA under different experimental $\mathrm{pH}$ conditions and following pre-saturation of ns-nHA with calcium. We hypothesized that should the carboxyl interaction with ns-nHA surface calcium ions be the dominant attachment mechanism, the more carboxyl groups provided by the amino acid and the lower the reaction $\mathrm{pH}$, the greater the coverage of ns-nHA with the amino acids. Similarly, by including a ns-nHA pre-saturation step with divalent calcium ions, we also hypothesized that functionalization of ns-nHA would be enhanced with dependence on initial reaction $\mathrm{pH}$. The pre-saturation of the as-received ns-nHA with calcium will also allow for the observation of an assumed stoichiometry impact of the nHA surface on amino acid adsorption.

\section{Results}

Confirming the Particle Size and Ca/P ratio of As-received and Unmodified ns-nHA. XRD analysis of our starting, dry powders reported a crystallinity of $66 \%$ following the approach by Del Valle et al. ${ }^{19}$, while BET (Brunauer, Emmett and Teller) analysis revealed a surface area of $89.91 \mathrm{~m}^{2} / \mathrm{g}$. In addition, scanning electron microscopy (SEM) confirmed that the particles were of rod-like shape (see Supplementary Information, Fig. S1), while from SEM with energy dispersive $\mathrm{x}$-ray (SEM-EDX) analysis the molar ratio of $\mathrm{Ca} / \mathrm{P}$ was found to be 1.52 and the ns-nHA was confirmed as being carbonated (see Supplementary Information, Fig. S2). SEM analysis also revealed that the ns-nHA particles were $\sim 120 \mathrm{~nm}$ in length and $20-30 \mathrm{~nm}$ in width on average.

Zeta Potential of ns-nHA becomes Less Negative upon Amino Acid Functionalization. Funct ionalization of the ns-nHA surface using amino acids resulted in changes to the local surface charge due to the presence of unbound $\mathrm{COO}^{-}$and $\mathrm{NH}_{2}-\mathrm{H}^{+}$groups from the amino acids, as well as differences in the amounts of vacant grafting sites on the ns-nHA surface (Fig. 1).

A three factor general linear model indicated that the interaction between reaction $\mathrm{pH}$ and both calcium saturation and amino acid type (both $\mathrm{p}<0.0001$ ), as well as between calcium saturation and amino acid type $(\mathrm{p}=0.001)$ was statistically detectable for zeta potential. In addition, the pre-saturation of ns-nHA with calcium and amino acid type were each independently found to impact zeta potential (both $\mathrm{p}<0.0001$ ). At stable reaction conditions ( $\mathrm{pH} 7.8$ and no pre-saturation with calcium), the addition of amino acids to the ns-nHA surface 


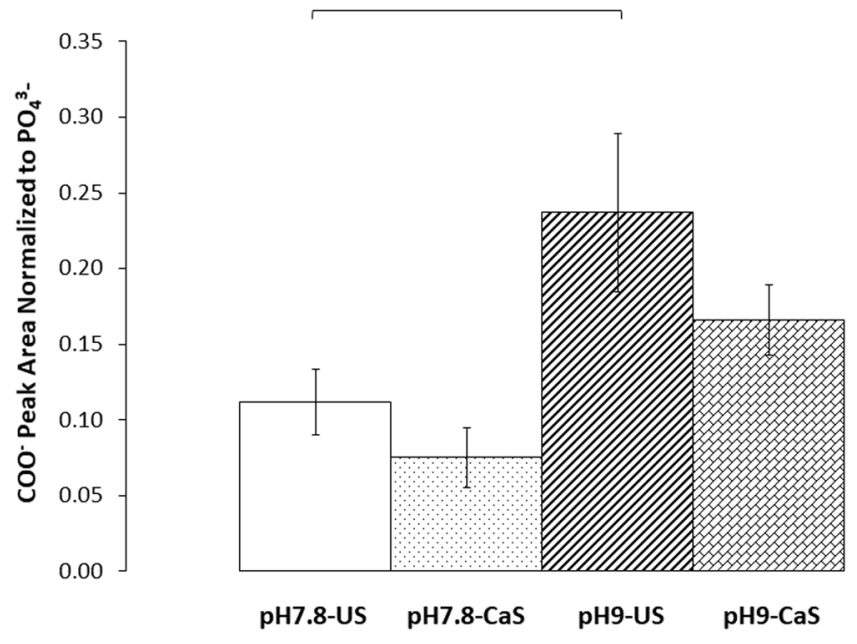

Figure 2. ATR-FTIR analysis of $\mathrm{COO}^{-}$peak area normalized to $\mathrm{PO}_{4}{ }^{3-}$ peak area for blank ns-nHA. Increasing the reaction $\mathrm{pH}$ notably increased the ratio of $\mathrm{COO}^{-}$symmetric stretch peak area to $\mathrm{PO}_{4}{ }^{3-}$ vibration peak area. The saturation of ns-nHA with calcium $(\mathrm{CaS})$ did not result in a detectably different $\mathrm{COO}^{-}$peak area compared to unsaturated (US). Data reported as average \pm one standard deviation $(n=3)$. Horizontal bars indicate a statistically detectable difference $(\mathrm{p}<0.05)$.

notably increased the zeta potential value (less negative) compared to the blank (i.e. unfunctionalized) ns-nHA. Furthermore, under calcium saturated and $\mathrm{pH} 7.8$ conditions, the ns-nHA surface functionalized with aspartic acid had the greatest zeta potential of all sample groups. With a near-neutral surface charge, there is minimal electrostatic repulsion between the like-charged aspartic acid-functionalized particles. A more balanced aspartic acid layer on the ns-nHA surface, with carboxyl and amine groups of the amino acid both serving as binding agents, likely contributed to this reported zeta potential.

ATR-FTIR COO- Symmetric Stretch Peak Area Greatest for Aspartic Acid, while $\mathrm{NH}_{2}-\mathrm{H}^{+}$ Symmetric Stretch Peak Area Greatest for Lysine. ATR-FTIR analysis revealed further differences in the amino acid-dependent affinity to the ns-nHA surface (see Supplementary Information, Fig. S3, for a representative stacked plot). The results of $\mathrm{COO}^{-}$peak area normalized to the $605 \mathrm{~cm}^{-1} \mathrm{PO}_{4}{ }^{3-}$ vibration peak for the blank ns-nHA groups are given in Fig. 2. Meanwhile, the normalized $\mathrm{COO}^{-}$and $\mathrm{NH}_{2}-\mathrm{H}^{+}$symmetric stretch peak areas (with that of the blank ns-nHA subtracted, respectively) are given for the amino acid functionalized ns-nHA in Figs 3 and 4.

For the blank ns-nHA samples, reaction $\mathrm{pH}$ was found to have a statistically detectable impact on $\mathrm{COO}^{-}$peak area $(\mathrm{p}=0.0004)$ using a two-way ANOVA. A contributing factor to the increase in normalized peak area is the likely reduced amount of phosphate species present for the blank ns-nHA as a result of hydrolysis during the experiment. This also corresponds to the higher (less negative) zeta potential observed for $\mathrm{pH} 9$ conditioned blank ns-nHA samples (Fig. 1) - having fewer phosphate species would reduce the amount of negative surface charge. Analysis of the $\mathrm{pH}$ change during reaction and characterization of the specific surface area (SSA) of the blank following functionalization supports the greater hydrolysis of ns-nHA at $\mathrm{pH} 9$ (see Figs S4 and S5, respectively, in supplementary information). For example, the change in $\mathrm{pH}$ during reaction is greater for the blank ns-nHA at $\mathrm{pH} 9$ than at $\mathrm{pH} 7.8$.

A three-factor general linear model of the $\mathrm{COO}^{-}$peak area data revealed that the interaction between amino acid type and pre-saturation of ns-nHA with calcium was statistically detectable $(\mathrm{p}=0.043)$, while $\mathrm{pH}$ $(\mathrm{p}<0.001)$, pre-saturation of ns-nHA with calcium $(\mathrm{p}<0.0001)$, and amino acid type $(\mathrm{p}<0.0001)$ each independently affected the normalized $\mathrm{COO}^{-}$peak area. With twice as many carboxyl functional groups per aspartic acid molecule, it is congruent that the $\mathrm{COO}^{-}$peak area for aspartic acid is almost twice as great as the other two amino acids (which only have one carboxyl functional group per molecule). In addition, for aspartic acid only, the pre-saturation of ns-nHA with calcium notably increased the $\mathrm{COO}^{-}$peak area; however, upon normalization for the number of carboxyl groups per aspartic acid molecule this effect is largely eliminated. Meanwhile, this same statistical model indicated that only the amino acid type impacted the normalized $\mathrm{NH}_{3}{ }^{+}$peak area $(\mathrm{p}<0.0001)$. Here it is important to note that there are twice as many amine functional groups per amino acid molecule for lysine as glycine. As a result, with $\mathrm{NH}_{2}-\mathrm{H}^{+}$peak areas almost twice as high for lysine as glycine, this then suggests that there was a similar amount of attachment to ns-nHA for these two amino acids (this is similarly supported by $\mathrm{COO}^{-}$peak area).

Amino Acid Attachment to ns-nHA Occurs via Both Carboxyl and Amine Functional groups. The impact of the different reaction conditions on amino acid affinity for the ns-nHA surface was also quantified using a ninhydrin protocol ${ }^{20}$ which characterizes the amine groups free at the ns-nHA surface, and a fluoraldehyde method ${ }^{21}$ which involves the digestion of ns-nHA and subsequent accounting of all amine groups present with ns-nHA (attached or free at the ns-nHA surface). The fraction of moles of amino acid attached to moles of 


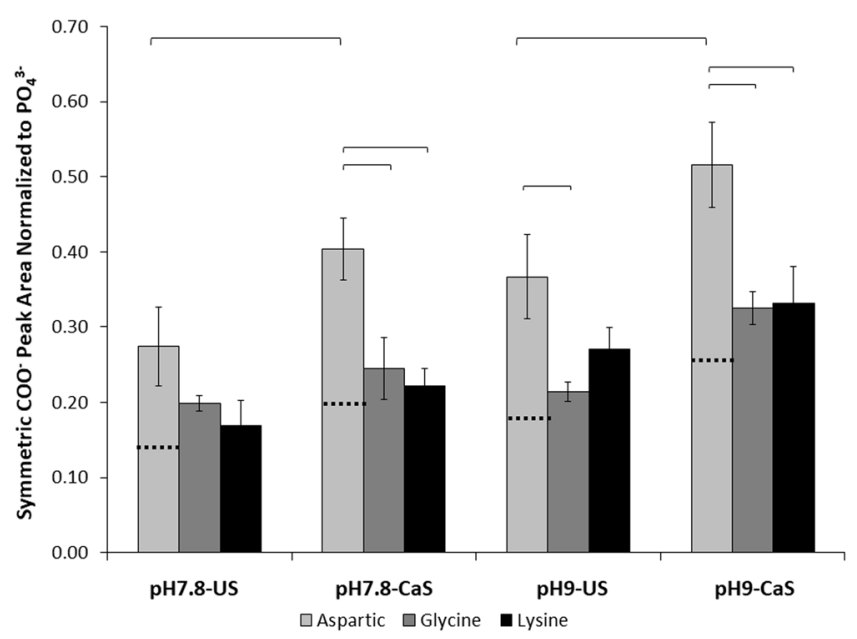

Figure 3. ATR-FTIR analysis of the amino acid functionalized ns-nHA COO${ }^{-}$peak area normalized to $\mathrm{PO}_{4}{ }^{3-}$ peak area and peak area of blank ns-nHA subtracted for each condition. Aspartic acid-functionalized ns-nHA was found to have the greatest symmetric $\mathrm{COO}^{-}$peak area. However, there are two $\mathrm{COO}^{-}$groups per aspartic acid molecule (glycine and lysine only have one $\mathrm{COO}^{-}$group per molecule). As shown by the black horizontal dashed line on the aspartic acid column, the $\mathrm{COO}^{-}$peak area analysis suggests that there is a comparable amount of aspartic acid attached to the ns-nHA surface as achieved with the other amino acids. Data reported as average \pm one standard deviation $(\mathrm{n}=3)$. ns-nHA unsaturated with calcium is given as US, while that presaturated with calcium is given as CaS. Horizontal bars indicate statistically detectable difference $(\mathrm{p}<0.05)$.

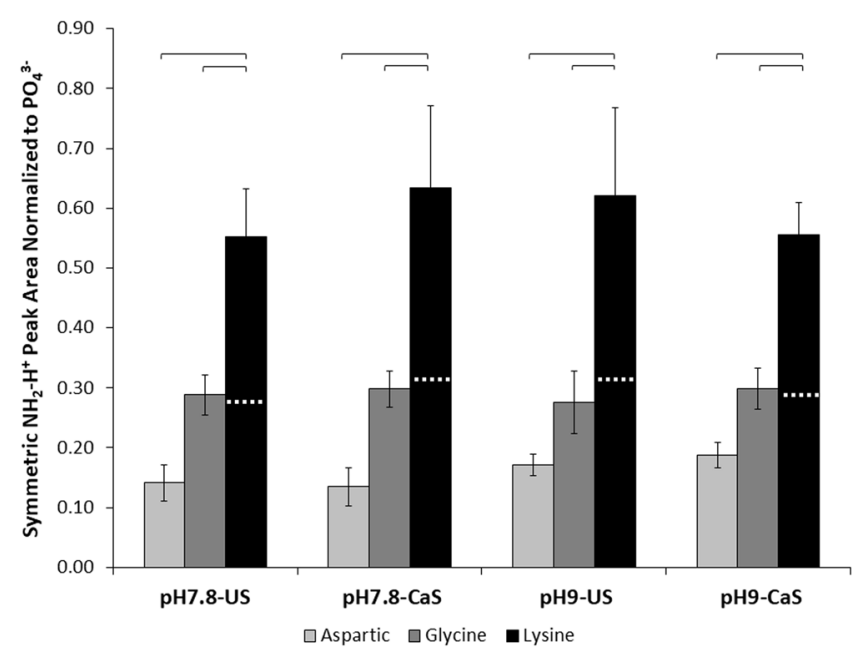

Figure 4. ATR-FTIR analysis of the amino acid functionalized ns-nHA $\mathrm{NH}_{2}-\mathrm{H}^{+}$peak area normalized to $\mathrm{PO}_{4}{ }^{3-}$ peak area. Lysine-functionalized ns-nHA was found to have the greatest symmetric $\mathrm{NH}_{2}-\mathrm{H}^{+}$peak area. However, there are two $\mathrm{NH}_{2}-\mathrm{H}^{+}$groups per lysine molecule (aspartic acid and glycine only have one $\mathrm{NH}_{2}-\mathrm{H}^{+}$ group per molecule). As shown by the white horizontal dashed line on the lysine column, the $\mathrm{NH}_{2}-\mathrm{H}^{+}$peak area analysis suggests that there is a comparable amount of lysine attached to the ns-nHA surface as glycine. Data reported as average \pm one standard deviation $(n=3)$. ns-nHA unsaturated with calcium is given as US, while that pre-saturated with calcium is given as CaS. Horizontal bars indicate statistically detectable difference $(\mathrm{p}<0.05)$.

sites originally available for grafting on ns-nHA is shown in Fig. 5 for the ninhydrin protocol and Fig. 6 for the fluoraldehyde method.

A three-factor general linear model analysis of the ninhydrin protocol data revealed that the interaction of reaction $\mathrm{pH}$ and amino acid type is detectably different $(\mathrm{p}<0.0001)$. Meanwhile, reaction $\mathrm{pH}(\mathrm{p}<0.0001)$, pre-saturation of ns-nHA with calcium $(\mathrm{p}=0.036)$, and amino acid type $(\mathrm{p}<0.0001)$ were each found to independently impact the fraction of sites grafted. As a result, only lysine sees a detectable increase in attachment to ns-nHA as $\mathrm{pH}$ increases. A similar model analysis of the fluoraldehyde assay data also indicated that the interaction of reaction $\mathrm{pH}$ and amino acid type was detectable $(\mathrm{p}=0.004)$. Furthermore, and as with the ninhydrin protocol, reaction $\mathrm{pH}(\mathrm{p}<0.0001)$, pre-saturation of ns-nHA with calcium $(\mathrm{p}=0.009)$, and amino acid type $(p<0.0001)$ were each found to independently impact the fraction of sites grafted as per the fluoraldehyde assay. 


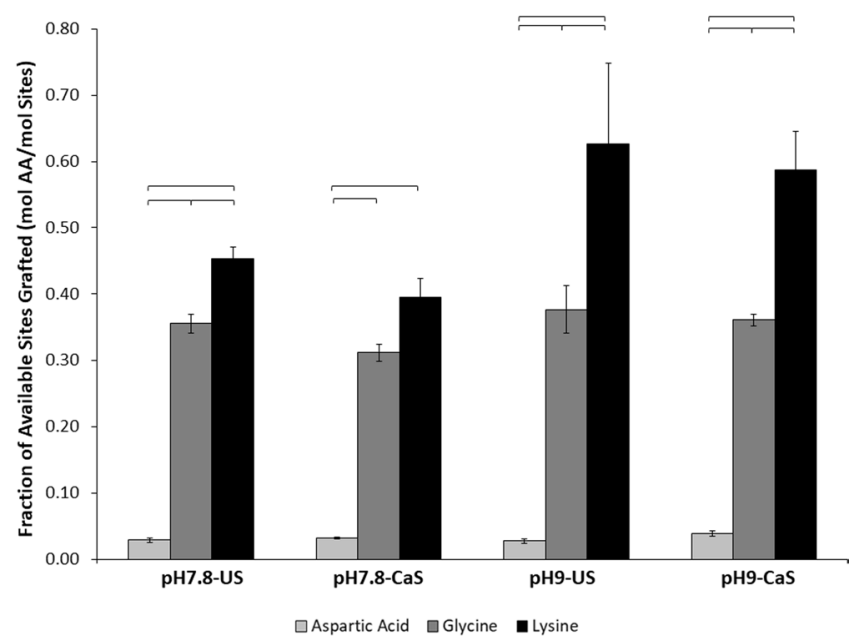

Figure 5. Molar ratio of total sites grafted on the ns-nHA surface using different amino acids (AA) following the ninhydrin protocol. Aspartic acid attached to fewer available graft sites on ns-nHA than glycine or lysine; this is likely indicative of the more balanced layer of aspartic acid on the ns-nHA surface. Data reported as average \pm one standard deviation $(n=5)$. ns-nHA unsaturated with calcium is given as US, while that presaturated with calcium is given as CaS. Horizontal bars indicate statistically detectable difference $(\mathrm{p}<0.05)$.

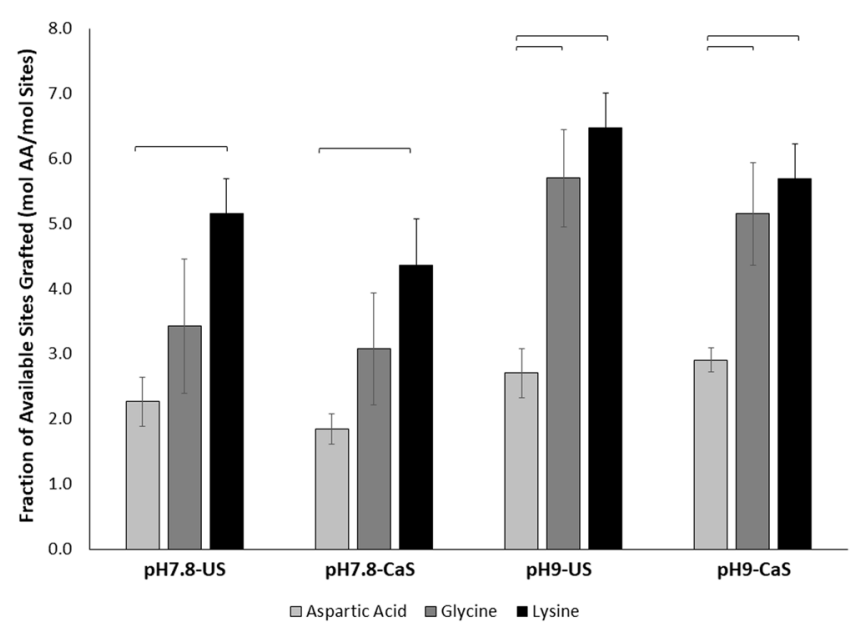

Figure 6. Molar ratio of total sites grafted on the ns-nHA surface using different amino acids (AA) following the fluoraldehyde protocol. Aspartic acid was again found to attach to fewer available graft sites on ns-nHA than the other two amino acids. Data reported as average \pm one standard deviation $(n=5)$. ns-nHA unsaturated with calcium is given as US, while that pre-saturated with calcium is given as CaS. Horizontal bars indicate statistically detectable difference $(\mathrm{p}<0.05)$.

The trend in the ratio of moles of amino acid detected to sites originally available for grafting on the surface of ns-nHA is similar for the ninhydrin and fluoraldehyde protocols. It is important to recognize that the use of two different protocols to quantify amino acid attachment to nHA limits the ability to compare pre- and post-digestion assay results with emphasis on type of amino acid and/or reaction condition. Yet, as any amine groups attached directly to the ns-nHA surface are not free to react until the ns-nHA is digested, the difference between the two protocols still gives some indication of the relative amount of amine groups involved in such an interaction. Altogether, the data suggests that the attachment of aspartic acid to the ns-nHA was more balanced and sparse than the other two amino acids studied.

\section{Discussion}

Crystalline $\mathrm{HA}$ is hexagonally close packed with its unit cell consisting of $\mathrm{Ca}^{2+}, \mathrm{PO}_{4}{ }^{3-}$ and $\mathrm{OH}^{-}$groups closely packed together in a hexagonal arrangement ${ }^{19,22}$. While $\mathrm{OH}$ serves as the backbone, the 6 phosphates helically arranged around the c-axis are responsible for being the skeletal frame that stabilizes $\mathrm{HA}^{22}$. With a $\mathrm{Ca} / \mathrm{P}$ ratio lower than the stoichiometric value for HA (i.e. 1.67) there are calcium vacancies on the surface of our ns-nHA. Assuming the substitution of tetrahedral phosphate with a planar carboxylate ion during the fabrication of nHA, the negative charge will have been reduced (i.e. increased zeta potential) and fewer calcium ions then necessary 
to balance the charge. As a result, the surface calcium atoms are likely covered by excess phosphate ions which, when protonated during pre-conditioning in distilled water, will produce $\mathrm{P}$-OH groups ${ }^{9,23,24}$. Particles of high surface area, such as found with nHA, are more likely to exhibit this thermodynamically stable surface deficiency in calcium, as a result of a dissolution and re-precipitation mechanism ${ }^{25}$. An additional characteristic of the as-received, unmodified ns-nHA is its low crystallinity and, as a result, some heterogeneity at the surface is expected. However, bone itself has a mineral phase crystallinity of $51-59 \%{ }^{26}$ and as a result of our interests in utilizing the surface-modified nHA in bone-mimicking composites, ns-nHA is found to be acceptable in the investigation presented here.

Blank ns-nHA (i.e. unfunctionalized) controls under regular experimental conditions (i.e. $\mathrm{pH} 7.8$ and unsaturated) are negatively charged as a result of calcium vacancies and the acidic $\mathrm{pK}_{\mathrm{a}}$ of the ns-nHA surface ${ }^{2,9}$. By pre-saturating the blank ns-nHA with calcium, the zeta potential becomes less negative, while increasing $\mathrm{pH}$ also makes this value less negative (although to a lesser degree than calcium saturation). The latter observation may be explained by the relative instability of the ns-nHA surface while held at $\mathrm{pH} 9$ and potential loss of phosphate groups (and/or increased calcium ion retention). The zeta potential dependence on the type of amino acid at the ns-nHA surface may be explained by a "cone" of adsorbance ${ }^{27}$ and greater fraction of unbound calcium upon carboxyl group attachment (for aspartic acid), compared to amine group attachment (for glycine and lysine). The lack of difference between zeta potential values for glycine- and lysine-functionalized ns-nHA may be explained by a slightly more disordered layer attachment of these two amino acids (compared to aspartic acid). Meanwhile, the impact of calcium saturation on zeta potential can be explained by increased repulsion between divalent calcium on the ns-nHA surface and amine groups of the amino acids, particularly for lysine which has a net positive charge. Overall, the change in zeta potential observed upon amino acid absorbance on ns-nHA surface is a result of the complex balance of phosphate ion loss, differences in calcium ion retention, and the additional charge associated with amino acid terminus and side groups. Furthermore, the degree to which the carboxyl and amine functional groups are ionized at a given $\mathrm{pH}$ will also impact attachment and resultant zeta potential of the functionalized particles. For example, while carboxyl groups are essentially all negatively charged at both $\mathrm{pH}$ conditions studied, less than $80 \%$ of the amine groups on aspartic acid and glycine are positively charged at $\mathrm{pH} 9$. Meanwhile, for lysine the alpha-amine group $\left(\mathrm{pK}_{\mathrm{a}}\right.$ of 8.95$)$ is less than $50 \%$ ionized at $\mathrm{pH} 9$. The fact that $\mathrm{pH}$ alone was not found to have a significant impact on zeta potential indicates the likely impact of not only heterogeneity at the ns-nHA surface prior to modification, but also the multi-modal attachment of amino acids to the surface (i.e. not solely governed by either the charged carboxyl or amine groups).

The significant increase in the normalized $\mathrm{COO}^{-}$peak area for blank ns-nHA upon increasing $\mathrm{pH}$ can be explained by the degradation of ns-nHA and loss of the phosphate anion, particularly in the absence of amino acids. The ATR-FTIR peak area analysis suggests that the factor of greatest impact on amino acid functionalization of ns-nHA in this study was the type of amino acid itself. In addition, the greater normalized $\mathrm{COO}^{-}$and $\mathrm{NH}_{2}-\mathrm{H}^{+}$peak areas for glycine and lysine functionalized ns-nHA compared to that for aspartic acid (particularly once accounting for number of functional groups per mole of amino acid) is related to the relative fraction of available sites grafted. A limitation for this study results from the ATR-FTIR peak fitting analysis and the normalization of the data to the $\mathrm{PO}_{4}{ }^{3-}$ vibration peak. Owing to hydrolysis of the ns-nHA powder during the experiment, the amount of phosphate species present after the reaction likely differ between sample groups (with the presence of amino acids and buffer solution impacting the hydrolysis reaction). As a result, the normalized peak areas of $\mathrm{COO}^{-}$and $\mathrm{NH}_{2}-\mathrm{H}^{+}$of amino acid functionalized ns-nHA match the general trend of the ninhydrin and fluoraldehyde protocols, but not the difference in magnitude between sample groups. To overcome this limitation, the latter protocols are needed to give a more complete analysis of the success of our functionalization approach.

On the surface of ns-nHA, there are two main binding sites (termed C and P sites) ${ }^{18,28}$. The $\mathrm{C}$ site is a calcium-rich region which binds strongly to anionic groups such as $\mathrm{COO}^{-}$, while the $\mathrm{P}$ site is phosphate-rich and interacts with cationic groups such as $\mathrm{NH}_{3}{ }^{+}$. From our studies, we conclude that each amino acid predominantly binds to the $\mathrm{P}$ sites of our negatively charged ns-nHA as a result of strong hydrogen bonding; however, $\mathrm{C}$ sites also have a detectable and meaningful amount of binding via electrostatic interactions. With "water-reacted" nHA ${ }^{11}$ there will be a considerable number of $\mathrm{POH}$ functional groups (at the $\mathrm{P}$ sites); these will form particularly strong hydrogen bonds with the amine groups of the amino acids. Figure 7 provides a schematic for the dominant mode of interaction between the ns-nHA surface and the different amino acids.

The quantified coverage of available ns-nHA graft sites suggests an increased ease of approach of the more positively charged amino acid (i.e. lysine) toward the negatively charged ns-nHA surface under each reaction condition compared to the negatively charged amino acid (i.e. aspartic acid). Aspartic acid attachment was more limited partly due to both it and ns-nHA having net negative charges and some electrostatic repulsion likely in the bulk medium. In addition, the number of graft sites truly available for functionalization should also be discussed. For example, prior work by Garcia-Ramos et al. ${ }^{27}$ theorized that the effective volume of adsorption is best modelled by a cone, where any cation located within this cone projection on the surface could not function as an adsorption site. As the cross-sectional area of a $\mathrm{COO}^{-}$group has previously been reported by Shafei et al. ${ }^{23}$ to be as high as $20.5 \mathrm{~A}^{2}$ and that of $\mathrm{NH}_{3}$ found to be $7.0 \mathrm{~A}^{2}$, the cone projection is greater for amino acid attachment via the carboxyl groups. As a result of this 'cone', the number of sites truly available for aspartic acid attachment via carboxyl groups is less than would be theoretically predicted based on amine attachment. The type of amino acid may also impact the size of these respective cone projections ${ }^{9}$. In addition, the model used to calculate the theoretically expected number of grafting sites is based on a fully crystalline material ${ }^{20}$. As our ns-nHA is not fully crystalline, it is important to recognize that the ninhydrin and fluoraldehyde assays in effect present a scaled comparison of the success of different amino acid attachment to the surface of ns-nHA. The use of two different protocols to present a pre- and post-digestion comparison is another limitation of this study. However, the difference between the two protocols still gives some indication of the relative amount of amine groups involved in such an interaction. Meanwhile, it is interesting to note that the pre-saturation of ns-nHA with calcium does not greatly 

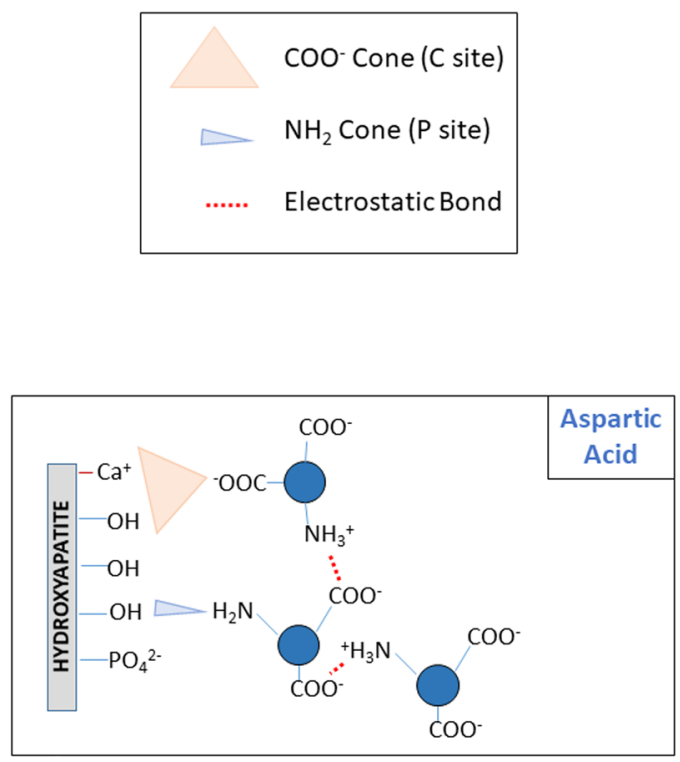
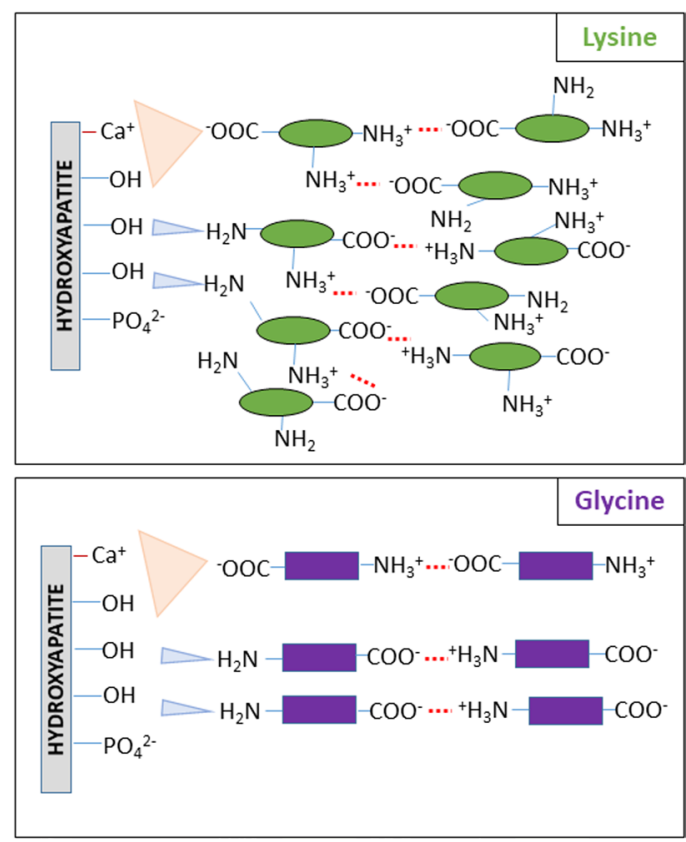

Figure 7. A proposed schematic for the dominant mode of adsorption of the different amino acids at the nsnHA surface. Both amine and carboxyl functional groups contributed to the attachment of the amino acids to the ns-nHA surface. This schematic generally encompasses each reaction condition, owing to little dependence observed on reaction condition (i.e. $\mathrm{pH}$ or pre-saturation with calcium); however, it is important to recognize that the fraction of amine groups which are ionized will change in the schematic as $\mathrm{pH}$ changes. Overall, the ease of approach towards the negatively charged ns-nHA surface is greatest for Lysine and Glycine, and more challenging for the negatively charged Aspartic acid. As a result, the attachment of Aspartic acid to the ns-nHA surface is more limited compared to the other two amino acids.

improve the attachment of any amino acid. This may be explained by a very small reduction in the negativity of the ns-nHA surface upon pre-saturation (as observed by zeta potential measurements). As a result, pre-saturation with calcium largely did not impact the ease with which amino acids may bind to the negatively charged ns-nHA via negatively charged carboxyl groups. Furthermore, as the fraction of total sites grafted in accordance with the post-digestion fluoraldehyde assay is more than $100 \%$ for each of the amino acids studied, it is likely that these amino acids attach in relatively thick layers at the ns-nHA surface. While prior investigations have reported that the affinity constants for amino acids are relatively weak ${ }^{2}$, our work suggests that the interaction between amino acid and the ns-nHA surface is notably mediated by the side group. With the molar ratio of amino acid to available ns-nHA sites for grafting being detectably lower than either glycine or lysine, the functionalization control is likely greatest with aspartic acid. In building a bone-mimicking composite, the use of aspartic acid for a more controlled and relatively stable layer functionalization of ns-nHA surface may be beneficial. Future investigation will consider reducing the ratio of amino acid to the ns-nHA in solution in order to improve the efficiency in the reaction and further limit the thickness of an amino acid layer on the ns-nHA surface.

\section{Conclusion}

In this study, we investigated the binding of three different amino acids for the surface of poorly crystalline, non-stoichiometric carbonated $\mathrm{nHA}$ (ns-nHA) under different experimental $\mathrm{pH}$ conditions and following pre-saturation of ns-nHA with calcium. The attachment affinity at the ns-nHA surface was found to be significantly greater for an amino acid with a basic (i.e. lysine) or non-polar side group (i.e. glycine) compared to an acidic side group (i.e. aspartic acid). This side group dependence was also influential on mode of amino acid attachment to the surface of ns-nHA. For example, both the amine and carboxyl groups significantly contributed to the attachment of the amino acids to ns-nHA; however, the relative proportion of amine group attachment differed depending on the amino acid. Furthermore, as the molar fraction of amino acids bound was greater than 1 following the fluoraldehyde assay, there appears to be a relatively thick layer of amino acid attaching on the ns-nHA surface. By manipulating the experimental conditions for ns-nHA surface functionalization, this study has notably improved the current understanding of amino acid affinity for the surface of nHA.

\section{Methods}

Characterization of As-Received ns-nHA Powder. Hydroxyapatite nanoparticles were purchased from MKNano (division of M K Impex Corp, Canada). To confirm the shape and size of the starting powder, scanning electron microscopy (SEM; Zeiss Merlin FESEM 1530) was performed, while SEM-energy dispersive x-ray analysis (SEM-EDX) was used to confirm powder composition. Prior to SEM analysis, powders were sputter coated in a magnetron sputtering machine for $120 \mathrm{~s}$ to a form an Au film on the particles. In addition, a 4-circle 
<smiles>NC(CC(O)C(=O)O)C(O)C(=O)O</smiles><smiles>NCCCCC(N)C(O)O</smiles>

8.95

Lysine

(LYS)

Figure 8. Molecular structure of Aspartic acid, Glycine, and Lysine with $\mathrm{pK}_{\mathrm{a}}$ values of amine and carboxyl groups represented near corresponding functional groups ( $\mathrm{pK}_{\mathrm{a}}$ values from Nelson et al. ${ }^{31}$ ).

high-resolution x-ray diffractometer (XRD; PANalytical Xpert Pro MRD) was used to confirm ns-nHA crystallinity, and BET (Brunauer, Emmett and Teller) analysis was used to characterize the surface area of the powder.

Amino Acid-Functionalization of ns-nHA Powder. The amino acid reagents used in this study were L-aspartic acid, glycine, and L-lysine mono-hydrochloride (all purchased from Sigma-Aldrich Canada Co). The three amino acids were selected based on the differences in their side chains and net charges under reaction conditions. For example, the $\mathrm{pI}$ of glycine is 5.97, of lysine is 9.74 , and of aspartic acid is $2.77^{2}$. Figure 8 depicts the molecular structure and $\mathrm{pK}_{\mathrm{a}}$ of the amine and carboxyl groups for these three amino acids.

The side chain group of glycine $(-\mathrm{H})$ is non-polar, while that of aspartic acid $\left(-\mathrm{CH}_{2} \mathrm{COO}^{-}\right)$is acidic and of lysine $\left(-\mathrm{CH}_{2} \mathrm{CH}_{2} \mathrm{CH}_{2} \mathrm{CH}_{2} \mathrm{NH}_{3}{ }^{+}\right)$is basic. As a result, at a reaction $\mathrm{pH}$ of 7.8 , lysine has a net positive charge, the aspartic acid has a net negative, and glycine is slightly negative. At a reaction $\mathrm{pH}$ of 9.0, the net charges remain similar in sign, but slightly more negative in magnitude in each case. Stock solutions of glycine and lysine at a concentration of $0.425 \mathrm{M}$ were prepared by adding the requisite mass of powder in $500 \mathrm{~mL}$ distilled water $\left(\mathrm{dH}_{2} \mathrm{O}\right)$. Meanwhile, as aspartic acid is minimally soluble in $\mathrm{dH}_{2} \mathrm{O}^{29}$, its $0.425 \mathrm{M}$ stock solution was prepared by adding the requisite amount of powder to $200 \mathrm{~mL}$ of $2.0 \mathrm{M}$ sodium hydroxide solution $\left(\mathrm{NaOH}_{(\mathrm{aq})}\right)$ until dissolved, and then toping the volume to $500 \mathrm{~mL}$ with $\mathrm{dH}_{2} \mathrm{O}$. The amino acid solutions were stored at room temperature until use.

To ensure the saturation of any calcium deficiencies on the hydroxyapatite surface and decrease the net negativity of the surface charge, $12 \mathrm{~g}$ of ns-nHA powder was added to $300 \mathrm{~mL}$ of $5.0 \mathrm{mM} \mathrm{CaCO}$ solution and placed on a horizontal shaker at $200 \mathrm{rpm}$ for 3 days. This ns-nHA powder was subsequently termed "CaS" (or calcium saturated). In order to match these pre-conditioning steps without the addition of further calcium, ns-nHA was conditioned under regular unsaturated conditions (labelled "US") prior to amino acid addition. Under these regular conditions, $12 \mathrm{~g}$ of ns-nHA powder was added to $300 \mathrm{~mL}$ of $\mathrm{dH}_{2} \mathrm{O}$ on a horizontal shaker at $200 \mathrm{rpm}$ for 3 days. After 3 days of pre-conditioning the $\mathrm{pH}$ of the ns-nHA suspensions was recorded using a Accumet ${ }^{\circledR} \mathrm{AB} 150$ $\mathrm{pH}$ meter $\left(\mathrm{pH}_{\text {initial }}\right)$.

Amino acid solutions were then added to each respective container of ns-nHA-based suspension until a total volume of $600 \mathrm{~mL}$ was reached. The $\mathrm{pH}$ of the amino acid-ns-nHA suspension was maintained at either 7.8 (the previously determined 'stable' ns-nHA aqueous suspension $\mathrm{pH}$ ) or 9.0 using $1.0 \mathrm{M} \mathrm{NaOH}_{(\mathrm{aq})}$ and $1.0 \mathrm{M} \mathrm{HNO}_{3(\mathrm{aq})}$ ), during initial amino acid addition and for an additional $15 \mathrm{~min}$. The container was then returned to the horizontal shaker at $200 \mathrm{rpm}$ for another 3 days. Following amino acid reaction with ns-nHA, the $\mathrm{pH}$ of the suspension was recorded using an Accumet ${ }^{\mathrm{TM}} \mathrm{AB} 150 \mathrm{pH}$ meter $\left(\mathrm{pH}_{\text {final }}\right)$, before the suspension was added to $50 \mathrm{~mL}$ conical tubes and centrifuged at $1200 \mathrm{rpm}$ for $5 \mathrm{~min}$. The powder was then collected following removal of the supernatant, and rinsed with $5 \mathrm{~mL}$ of fresh $\mathrm{dH}_{2} \mathrm{O}$ before being re-spun at $1200 \mathrm{rpm}$ for another $5 \mathrm{~min}$. The powder was collected after removing this last supernatant and then dried in a $37^{\circ} \mathrm{C}$ oven. The final dried powders were stored in glass scintillation vials at room temperature until needed for characterization. The change in $\mathrm{pH}$ as a result of the amino acid functionalization reaction was calculated as $\Delta \mathrm{pH}=\mathrm{pH}_{\text {initial }}-\mathrm{pH}_{\text {final. }}$. A positive $\Delta \mathrm{pH}$ indicates a decrease in $\mathrm{pH}$ during the 3 days of reaction.

Zeta Potential Characterization of Amino Acid-Functionalized ns-nHA. Following drying the surface charge and relative suspension stability of the amino acid-functionalized ns-nHA was assessed by characterizing the zeta potential of ns- $\mathrm{nHA}-\mathrm{dH}_{2} \mathrm{O}$ solutions with a zeta potential analyzer (Wallis ${ }^{\mathrm{TM}}$, Cordouan 
Technologies) and ZetaQ V1.7.0 software $(\mathrm{n}=5)$. Sample suspensions of $0.5 \mathrm{mg} / \mathrm{mL} \mathrm{ns}-\mathrm{nHA}$ were prepared in MilliQ $\mathrm{dH}_{2} \mathrm{O}^{30}$ and buffered to $\mathrm{pH} 9.0$ using $0.1 \mathrm{M} \mathrm{NaOH}$ or $0.1 \mathrm{M} \mathrm{HNO}_{3}$ were prepared prior to measurement. A $\mathrm{pH}$ of 9.0 was chosen following the approach by Gonzalez-McQuire et al. ${ }^{1}$ for zeta potential measurement of HA.

Specific Surface Area (SSA) Characterization of Amino Acid-Functionalized ns-nHA. Surface area measurements were performed on a Quantachrome Autosorb-iQ/MP using liquid nitrogen as the adsorbent gas. Samples were degassed at $250^{\circ} \mathrm{C}$ for 1.5 hours under nitrogen gas and backfilled with helium. BET surface area was determined by using a multi-point BET analysis using 12 adsorption points equally spaced from $0.025 \mathrm{P} /$ $\mathrm{P} 0$ to $0.3 \mathrm{P} / \mathrm{P} 0$.

ATR-FTIR Analysis of Amino Acid-Functionalized ns-nHA. The ns-nHA powder was also analyzed by attenuated total reflectance Fourier transform infrared (ATR-FTIR) spectroscopy (Tensor 27, Bruker, Germany) to confirm the adsorption of the three amino acids $(\mathrm{n}=3)$. From the ATR-FTIR spectra, vibrations corresponding to $\mathrm{PO}_{4}{ }^{3-}$ were identified at 1028,605 , and $565 \mathrm{~cm}^{-1}$, while bands indicating $\mathrm{COO}^{-}$and $-\mathrm{NH}_{2}-\mathrm{H}^{+}$ symmetric/asymmetric stretches were identified at $1400-1430 / 1560-1600 \mathrm{~cm}^{-1}$ and $1550-1485 / 1590-1660 \mathrm{~cm}^{-1}$, respectively ${ }^{1}$. The peak area for each of the aforementioned functional groups was calculated using DMFit 2010 Software. The peak areas for the $\mathrm{COO}^{-}$and $-\mathrm{NH}_{2}-\mathrm{H}^{+}$stretches were subsequently normalized to that of the phosphate vibration at $605 \mathrm{~cm}^{-1}$ followed by subtracting the matching peak for the blank ns-nHA in order to give an estimation of the relative amounts of amino acid adsorbed on the ns-nHA.

Pre- and Post- ns-nHA Digestion Assays for Amino Acid Attachment to ns-nHA. The success of amino acid adsorption was further quantified relative to the theoretical availability of grafting sites using both a pre-digestion ninhydrin colourimetric method and a post-digestion fluoraldehyde method. Firstly, the ninhydrin method involves the reaction of free amine groups with a ninhydrin-ethanol solution, and the formation of a Ruhemann's purple dye anion under basic conditions which can then be photometrically quantified ${ }^{20}$. Utilizing a modified version of an existing ninhydrin staining protocol by Poli et al. ${ }^{20}$, reference amine solutions at a concentration of $1.01 \times 10^{-3} \mathrm{M}$ were prepared using the original amino acid stock solutions and absolute ethanol, while a ninhydrin solution at $1.97 \times 10^{-3} \mathrm{M}$ in absolute ethanol was prepared. Calibration standard solutions were prepared by mixing 0.5 to $5.0 \mathrm{~mL}$ of the reference amine solution with $1 \mathrm{~mL}$ of the ninhydrin solution, and topping up the total volume of this standard to $6 \mathrm{~mL}$ with absolute ethanol. To $50 \mathrm{mg}$ of amino acid-functionalized ns-nHA powder in a $15 \mathrm{~mL}$ conical tube, $1 \mathrm{~mL}$ of ninhydrin solution and $5 \mathrm{~mL}$ of absolute ethanol were added. The tubes containing either control or sample were added to a hot water bath at $90^{\circ} \mathrm{C}$ for $90 \mathrm{~min}$. After $90 \mathrm{~min}$, the tubes were cooled to room temperature and centrifuged at $4000 \mathrm{rpm}$ for $10 \mathrm{~min}$. The supernatant from these tubes was then collected and the remaining powder dried overnight at $37^{\circ} \mathrm{C} .150 \mu \mathrm{L}$ of the supernatant was then added to 96-well plates in triplicate and the absorbance read at $586 \mathrm{~nm}$ using a SpectramaxPlus (Molecular Devices) and SoftMax Pro V5.4.1 software $(\mathrm{n}=5)$.

While the ninhydrin method allows for the detection of free amine groups of amino acids otherwise attached to the surface of ns-nHA, any amine groups unavailable (e.g. directly attached to ns-nHA) are not detected. As we suspect that carboxyl groups are not the only significant functional group involved in attaching amino acids to the ns-nHA and the ninhydrin protocol is more efficient at slightly basic conditions, another method is needed to free amino acids from ns-nHA and subsequently quantify their presence. To address this $10 \mathrm{mg}$ of ns-nHA powder was digested by adding $400 \mu \mathrm{L}$ of $4 \mathrm{~N}$ hydrochloric acid and vortexing for $30 \mathrm{~s}$. To this clear solution $2 \mathrm{~mL}$ of $\mathrm{MQ} \mathrm{dH}_{2} \mathrm{O}$ and $225 \mu \mathrm{L}$ of $4 \mathrm{~N} \mathrm{NaOH}$ were added to further dilute and increase the $\mathrm{pH}$ to $\sim 5$, respectively. Standard solutions were prepared in a range of concentrations for each amino acid studied $\left(4.25 \times 10^{-5}\right.$ to $\left.4.25 \times 10^{-2} \mathrm{M}\right)$. To a black Corning $^{\text {TM }} 96$-well plate $100 \mu \mathrm{L}$ of sample or control solution was added, with $150 \mu \mathrm{L}$ of Phthaldialdehyde reagent (OPA; Sigma-Aldrich, Canada) then added to each well immediately prior to fluorescence detection at $455 \mathrm{~nm}$ using an excitation wavelength of $340 \mathrm{~nm}$ with a plate reader (BioTek ${ }^{\circledR}$ Instruments) $(n=5)$.

The theoretical calculation for the amount of sites available for grafting was derived using Eq. $(1)^{20}$.

$$
\mathrm{n}_{\text {sites }}=\mathrm{SSA} *\left[8 /\left(6 * \mathrm{~A} * \mathrm{~N}_{\mathrm{a}}\right)\right]
$$

where SSA is the specific surface area of the ceramic $\left(\mathrm{m}^{2} / \mathrm{g}\right)$, A is the total area of the unit cell $\left(\mathrm{m}^{2}\right)$, and $\mathrm{N}_{\mathrm{a}}$ is Avogadro's number. Using the determined concentration of amino acid present on the ns-nHA ( $\mathrm{n}_{\text {actual }}$ ) surface and this theoretical number of available sites for grafting $\left(n_{\text {sites }}\right)$, the percentage of sites successfully grafted was calculated as $\left[\mathrm{n}_{\text {actual }} / \mathrm{n}_{\text {sites }}\right] \times 100 \%$. It is important to note that this Eq. (1) assumes perfect crystallinity with a regularly repeating unit cell, which is a significant assumption given the low crystallinity of our ns-nHA.

Statistical Analysis. Differences in the means of study outcomes were analyzed using IBM $^{\circledR}$ SPSS $^{\circledR}$ Statistics software and a two-way ANOVA or three-factor univariate general linear model with a significance value of $\mathrm{p}=0.05$. In addition, a post-hoc Tukey analysis was performed upon confirming data normality. All data is presented in this paper as mean $+/$ - one standard deviation.

Data availability. The datasets generated and analyzed during the current study are available from the corresponding author upon reasonable request.

\section{References}

1. Gonzalez-McQuire, R., Chane-Ching, J. Y., Vignaud, E., Lebugle, A. \& Mann, S. Synthesis and characterization of amino acidfunctionalized hydroxyapatite nanorods. J. Mater. Chem. 14, 2277-2281 (2004).

2. Jack, K. S., Vizcarra, T. G. \& Trau, M. Characterization and Surface Properties of Amino-Acid-Modified Carbonate-Containing Hydroxyapatite Particles. Langmuir 12233-12242, https://doi.org/10.1021/la701848c (2007). 
3. Ivanova, T. I., Frank-Kamenetskaya, O. V., Kol'tsov, A. B. \& Ugolkov, V. L. Crystal structure of calcium-deficient carbonated hydroxyapatite. Thermal decomposition. J. Solid State Chem. 160, 340-349 (2001).

4. Lee, W.-H., Loo, C.-Y. \& Rohanizadeh, R. A review of chemical surface modification of bioceramics: effects on protein adsorption and cellular response. Colloids Surf. B. Biointerfaces 122, 823-34 (2014).

5. Koutsopoulos, S. \& Dalas, E. Hydroxyapatite crystallization in the presence of amino acids with uncharged polar side groups: glycine, cysteine, cystine, and glutamine. Langmuir 17, 1074-1079 (2001).

6. Koutsopoulos, S. \& Dalas, E. The Crystallization of Hydroxyapatite in the Presence of Lysine. J. Colloid Interface Sci. 231, 207-212 (2000).

7. Koutsopoulos, S. \& Dalas, E. Inhibition of hydroxyapatite formation in aqueous solutions by amino acids with hydrophobic side groups. Langmuir 16, 6739-6744 (2000).

8. Koutsopoulos, S. \& Dalas, E. Hydroxyapatite crystallization in the presence of serine, tyrosine and hydroxyproline amino acids with polar side groups. J. Cryst. Growth 216, 443-449 (2000).

9. Jahromi, M. T., Yao, G. \& Cerruti, M. The importance of amino acid interactions in the crystallization of hydroxyapatite. J. R. Soc. Interface 10, 20120906 (2013).

10. Koutsopoulos, S. \& Dalas, E. The effect of acidic amino acids on hydroxyapatite crystallization. J. Cryst. Growth 217, 410-415 (2000).

11. Rimola, A., Corno, M., Garza, J. \& Ugliengo, P. Ab initio modelling of protein-biomaterial interactions: Influence of amino acid polar side chains on adsorption at hydroxyapatite surfaces. Philos. Trans. R. Soc. A Math. Phys. Eng. Sci. 370, 1478-1498 (2012).

12. Sharma, R., Pandey, R. R., Gupta, A. A., Kar, S. \& Dhayal, M. In situ amino acid functionalization and microstructure formation of hydroxyapatite nanoparticles synthesized at different $\mathrm{pH}$ by precipitation route. Mater. Chem. Phys. 133, 718-725 (2012)

13. Pan, H., Tao, J., Xu, X. \& Tang, R. Adsorption Processes of Gly and Glu Amino Acids on Hydroxyapatite.pdf. Langmuir 23, $8972-8981$ (2007).

14. Zhai, Y. \& Cui, F. Z. Recombinant human-like collagen directed growth of hydroxyapatite nanocrystals. J. Cryst. Growth 291, 202-206 (2006).

15. Palazzo, B. et al. Amino acid synergetic effect on structure, morphology and surface properties of biomimetic apatite nanocrystals. Acta Biomater. 5, 1241-1252 (2009).

16. Matsumoto, T. et al. Crystallinity and solubility characteristics of hydroxyapatite adsorbed amino acid. Biomaterials 23, 2241-2247 (2002).

17. Ogata, S., Umeno, Y. \& Kohyama, M. First-principles approaches to intrinsic strength and deformation of materials: perfect crystals, nano-structures, surfaces and interfaces. Model. Simul. Mater. Sci. Eng. 17, 13001 (2009).

18. Lee, W. H., Loo, C. Y., Zavgorodniy, A. V. \& Rohanizadeh, R. High protein adsorptive capacity of amino acid-functionalized hydroxyapatite. J. Biomed. Mater. Res. - Part A 101 A, 873-883 (2013).

19. del Valle, L. J. et al. DNA adsorbed on hydroxyapatite surfaces. J. Mater. Chem. B 2, 6953-6966 (2014).

20. Poli, E. et al. Efficient quantification of primary amine functions grafted onto apatite ceramics by using two UV-Vis spectrophotometric methods. Anal. Methods 6, 9622-9627 (2014).

21. Loessner, D. et al. Functionalization, preparation and use of cell-laden gelatin methacryloyl-based hydrogels as modular tissue culture platforms. Nat. Protoc. 11, 727-746 (2016).

22. Poralan, G. M., Gambe, J. E., Alcantara, E. M. \& Vequizo, R. M. X-ray diffraction and infrared spectroscopy analyses on the crystallinity of engineered biological hydroxyapatite for medical application. IOP Conf. Ser. Mater. Sci. Eng. 79, 12028 (2015).

23. El Shafei, G. M. S. \& Moussa, Na Adsorption of Some Essential Amino Acids on Hydroxyapatite. J. Colloid Interface Sci. 238, $160-166(2001)$

24. Sarig, S. Aspartic acid nucleates the apatite crystallites of bone: a hypothesis. Bone 35, 108-13 (2004).

25. Brown, P. W. \& Martin, R. I. An Analysis of Hydroxyapatite Surface Layer Formation. J. Phys. Chem. B 103, 1671-1675 (1999),

26. Grynpas, M. The crystallinity of bone mineral. J. Mater. Sci. 11, 1691-1696 (1976).

27. Garcia-Ramos, J. V., Carmona, P. \& Hidalgo, A. The adsorption of acidic amino acids and homopolypeptides on hydroxyapatite. $J$. Colloid Interface Sci. 83, 479-484 (1981)

28. Spanos, N., Klepetsanis, P. G. \& Koutsoukos, P. G. Model Studies on the Interaction of Amino Acids with Biominerals: The Effect of L-Serine at the Hydroxyapatite-Water Interface. J. Colloid Interface Sci. 236, 260-265 (2001).

29. Dalton, J. \& Schmidt, C. The solubilities of certain amino acids in water, the densities of their solutions at twenty-five degreese, and the calculated heats of solution and partial molal volumes. J. Biol. Chem. 103, 549-578 (1933).

30. Hui, Y. et al. Preparation and application of hydroxyapatite (HA) nanoparticles/NR2B-siRNA complex. Trans. Nonferrous Met. Soc. China 18, 913-918 (2008).

31. Nelson, D. L. \& Cox, M. M. Principles of Biochemistry. 17, (Springer-Verlag, 2001)

\section{Acknowledgements}

The authors would like to thank the Natural Science and Engineering Research Council of Canada for their support in the form of a postdoctoral fellowship (P.C.) and a Discovery Grant (T.W.). This study was also partially supported by the Canadian Institutes of Health Research (Institute for Musculoskeletal Health and Arthritis) New Investigator Bridge Funding (T.W.) and the Dean of Engineering at the University of Waterloo (T.W.). The authors would also like to acknowledge Andrew Kacheff for collecting SSA data.

\section{Author Contributions}

P.C. conceived and conducted the experiments, analyzed and interpreted the results, and was the primary author of the manuscript. T.W. acquired the research operating funding, advised on the conduction of the experiments and data analysis, and assisted in the editing of the manuscript.

\section{Additional Information}

Supplementary information accompanies this paper at https://doi.org/10.1038/s41598-018-31058-5.

Competing Interests: The authors declare no competing interests.

Publisher's note: Springer Nature remains neutral with regard to jurisdictional claims in published maps and institutional affiliations. 
(i) Open Access This article is licensed under a Creative Commons Attribution 4.0 International License, which permits use, sharing, adaptation, distribution and reproduction in any medium or format, as long as you give appropriate credit to the original author(s) and the source, provide a link to the Creative Commons license, and indicate if changes were made. The images or other third party material in this article are included in the article's Creative Commons license, unless indicated otherwise in a credit line to the material. If material is not included in the article's Creative Commons license and your intended use is not permitted by statutory regulation or exceeds the permitted use, you will need to obtain permission directly from the copyright holder. To view a copy of this license, visit http://creativecommons.org/licenses/by/4.0/.

(C) The Author(s) 2018 